\title{
Inestabilidad microsatelital en lesiones preneoplásicas y neoplásicas del cuello uterino. Correlación con el genotipo del virus papiloma humano
}

\author{
Juan Carlos Roa $\mathbf{S}^{1}$, Ricardo Martínez $\mathbf{S}^{\mathbf{1}}$, Sonia Montenegro ${ }^{2}$, \\ Iván Roa $E^{1,4}$, Italo Capurro V3, G ilda Ibacache $S^{1}$, \\ Angélica Melo $\mathrm{A}^{\mathbf{1}}$. \\ Microsatellite instability \\ and human papilloma virus genotypes \\ in preneoplastic and neoplastic \\ uterine cervix lesions
}

Background: The association between some specific human papilloma virus (HPV) types and cervix cancer is well known. However, the genetic conditions that favor the development of cervical cancer are less well known. Aim: To determine the presence of satellite instability (MSI) in preneoplastic and neoplastic lesions of the cervix and correlate these findings with HPV genotypes. Material and methods: Biopsy samples of cervical lesions were studied. Sixteen had low grade lesions, 22 had high grade lesions and 28 had an epidermoid cancer. Viral types were identified with polymerase chain reaction, dot-blot hybridization and restriction fragment length polymorphism. MSI was determined using a panel of eight highly informative microsatellites. Results: Microsatellite instability in at least one locus was observed in 91, 56 and 69\% of low grade lesions, high grade lesions and epidermoid carcinomas, respectively. MSI-High grade, MSI-Low grade instability and microsatellite stability were observed in 5, 60 and $46 \%$ of samples, respectively. Two of three samples with high grade instability had HPV 52 genotype. Other viral subtypes had frequencies that ranged from $78 \%$ to $100 \%$, with the exception of HPV16 that was present in only $53 \%$ of samples with low grade instability. Conclusions: Two thirds of biopsy samples from cervical lesions had MSI, mechanism that can be involved in the first stages of cervical carcinogenesis. The low frequency of high grade instability, its association with HPV52 and the low frequency of HPV16 in samples with low grade instability, suggest different coadjutant mechanisms in cervical carcinogenesis (Rev Méd Chile 2007; 135: 37-44).

(Key words: Microsatellite repeats; Papillomavirus, human; Uterine cervical neoplasms)
Recibido el 7 de marzo, 2006. Aceptado el 19 de julio, 2006.
Abreviaciones
MSI-H: Inestabilidad microsatelital de alto grado.
MSI-L: Inestabilidad microsatelital de bajo grado.
MSS: Estabilidad microsatelital.
LBG: Lesión de bajo grado.
LAG: Lesión de alto grado.

Financiado por la Universidad de La Frontera a través del Proyecto DIUFRO № 120627.

${ }_{1}^{1}$ Departamento de Anatomía Patológica, Facultad de Medicina, Universidad de La Frontera. Temuco, Chile. ${ }^{2}$ Molecular Immunogenetics Laboratory. Ochsner Clinic Foundation, New Orleans, LA U.S.A. ${ }^{3}$ Departamento de Obstetricia y Ginecología, Facultad de Medicina, Universidad de La Frontera. Temuco, Chile. ${ }^{4}$ Departamento de Patología, Clínica Alemana Santiago, Chile.

Correspondencia a: Dr. Juan Carlos Roa. Departamento de Patología, Facultad de Medicina, Universidad de La Frontera. Manuel Montt 112. Código Postal 478-1176. Temuco, Chile. E mail: jcroa@ufro.cl 
$\mathrm{E}^{1}$ rol oncogénico del virus papiloma humano (HVP) ha sido claramente establecido en la carcinogénesis del cáncer cervical uterino $(\mathrm{CaCu})^{1,2}$. Sin embargo, la infección por sí misma, es insuficiente para la transformación del epitelio cervical, sugiriendo que otros factores como, la pérdida de la heterocigosidad $(\mathrm{LOH})$ o la inestabilidad de microsatélites (MSI), podrían estar involucrados en la tumorigénesis cervical ${ }^{3-5}$.

La MSI es un evento que ocurre en pequeños segmentos repetitivos de $\mathrm{ADN}$, denominados microsatélites, y la variación de estas regiones microsatélites es generada por errores durante la replicación del ADN. De esta manera, la MSI es un indicador de la incidencia elevada de mutaciones en el genoma de células neoplásicas, producto de la reparación defectuosa del $\mathrm{ADN}^{6}$. En el tejido normal, la presencia de MSI es infrecuente, debido a que cualquier desperfecto en la alineación de una hebra de ADN es corregido por el sistema reparador de ADN (Mismatch Repair System) $(\mathrm{MMR})^{7}$.

La MSI ha sido observada en una variedad de tumores como: colon $^{8}$, endometrio ${ }^{9}$, estómago ${ }^{10,11}$, páncreas ${ }^{12,13}$ y pulmón ${ }^{14,15}$. En tumores ginecológicos, la frecuencia de MSI ha sido reportada entre $17 \%$ y $30 \%$ para carcinoma endometrial $^{16-18}$ y entre $10 \%$ y $30 \%$ en carcinomas de ovario ${ }^{19-21}$. En $\mathrm{CaCu}$, la MSI ha sido observada entre $5 \%$ y $15 \% 3,9,22$ y alrededor de $15 \%$ en las neoplasias cervicales intraepiteliales 23 .

En relación a MSI y genotipos de HPV, tanto en LBG, LAG y carcinomas invasores, existe poca información. Por otro lado, la presencia de MSI en lesiones preneoplásicas o cánceres y su relación con el pronóstico de la patología queda aún por clarificarse.

Considerando lo anteriormente expuesto, nuestro objetivo general fue establecer una asociación entre subtipos específicos de HPV y la presencia de MSI en lesiones preneoplásicas y neoplásicas del cuello uterino.

\section{MATERIAL y MÉTOdos}

Muestras. Seleccionamos 66 muestras de archivo de cuello uterino (biopsias colposcópicas, LEEP y conizaciones) desde el archivo del Departamento de Anatomía Patológica de la Universidad de La
Frontera, obtenidas entre 1996 y 2001, las muestras provenían del Policlínico de Patología Cervical del Hospital Hernán Henríquez Aravena de Temuco, correspondientes a 16 lesiones de bajo grado (LBG), 22 lesiones de alto grado (LAG) y 28 carcinomas epidermoides.

Extracción del ADN. A 5 cortes de 10 micrones, se le microdisecó manualmente una zona con lesión y un área sin lesión, de acuerdo a lo indicado en un corte final teñido con hematoxilina-eosina. El ADN fue extraído con el Kit Puregene ${ }^{\mathrm{TM}}$ DNA Isolation System (Gentra Systems USA) siguiendo las instrucciones del fabricante. El ADN se almacenó a $-20^{\circ} \mathrm{C}$ hasta su análisis.

Para conocer la integridad del ADN y excluir muestras con ADN no amplificable, se utilizó una PCR con iniciadores para el gen ß-globina, que amplifican un fragmento de 268 pares de bases $(\mathrm{pb})^{24}$.

Tipificación viral. Para la detección del ADN viral se utilizaron dos sistemas de PCR de consenso dirigidos a los genes L1 y E6/E7. La tipificación se realizó por hibridación dot blot (Figura 1A) y RFLP (polimorfismo de fragmentos de restricción) (Figura $1 \mathrm{~B})$, según métodos publicados previamente ${ }^{25-28}$. Se consideró subtipos indeterminados a aquellos casos donde no se realizó RFLP y sólo fueron genotipificados mediante hibridación dot blot en tipos de AR o BR, pero no fue posible identificar el genotipo específico.

Marcadores microsatélites. Se utilizaron 8 marcadores dentro de los cromosomas 2, 3, 5, 12, 16 y 17. Un panel de 5 marcadores mononucleótidos y dinucleótidos (BAT25, BAT26, D2S123, D5S346 y D17S250) recomendados por el National Cancer Institute $(\mathrm{NCI})^{29}$ y 3 marcadores adicionales: D3S1067, D12S1638 y D16S265, elegidos por ser altamente polimórficos y por su alto rendimiento en material fijado en formalina e incluido en parafina.

Amplificación de microsatélites mediante PCR (PCR-MSI). La reacción de polimerasa en cadena (PCR) fue realizada con $5 \mu \mathrm{l}$ de ADN (5-10 ng) y volumen final de $15 \mu$ l. La mezcla de reacción fue: $0,2 \mathrm{uM}$ de partidores, $1,5 \mathrm{mM}$ de $\mathrm{MgCl}_{2}$ y $0,04 \mathrm{U} /$ ul de Taq polimerasa. Uno de los partidores fue marcado en su extremo terminal con deoxiadeno- 
sina trifosfato unido a fósforo gamma ${ }^{32}\left(\gamma \mathrm{P}^{32}\right)$ mediante polinucleótido quinasa T4. Las temperaturas de hibridación fueron optimizadas para cada marcador. Los productos PCR, tanto del área con lesión como del área sin lesión del mismo paciente, fueron corridos en paralelo sobre un gel denaturante (formamida-urea) al 12\%. Los geles fueron fijados en metanol al 10\% y ácido acético al $5 \%$ y lavados con agua desionizada. Posteriormente, se secaron al vacío a $80^{\circ} \mathrm{C}$ y expuestos a una placa autorradiográfica por 16 a 24 h a temperatura ambiente.

Detección de MSI. Cualquier cambio en la longitud de un microsatélite producido por inserción 0 deleción de un segmento de ADN repetitivo, mono o dinucleótido, en el tejido con lesión comparado con el tejido morfológicamente normal; observado como movilidad electroforética de las bandas en geles denaturantes al 12\% (Figura 1).
Según la frecuencia de MSI en los marcadores usados, se han definido 3 categorías de tumores: los de MSI de alta frecuencia (MSI-H), cuando más de $30 \%$ de los marcadores muestran MSI, los de baja frecuencia (MSI-L) cuando 30\% o menos de los marcadores están alterados y los tumores que no son afectados, llamados microsatélites estables (MSS) ${ }^{29}$.

\section{RESULTADOS}

La edad del grupo estudiado fluctuó entre 24 y 79 años, con una media de 39 años. Se recolectaron 16 LBG, 22 LAG y 28 casos de carcinoma epidermoide (Figura 2). Los subtipos de HPV 33, 16 y 31 fueron los más frecuentes, observándose casi exclusivamente en carcinomas y lesiones alto grado. Se observó un pequeño grupo de casos con infección mixta, la mayor parte de ellos correspondió a lesiones de alto grado con coinfec-

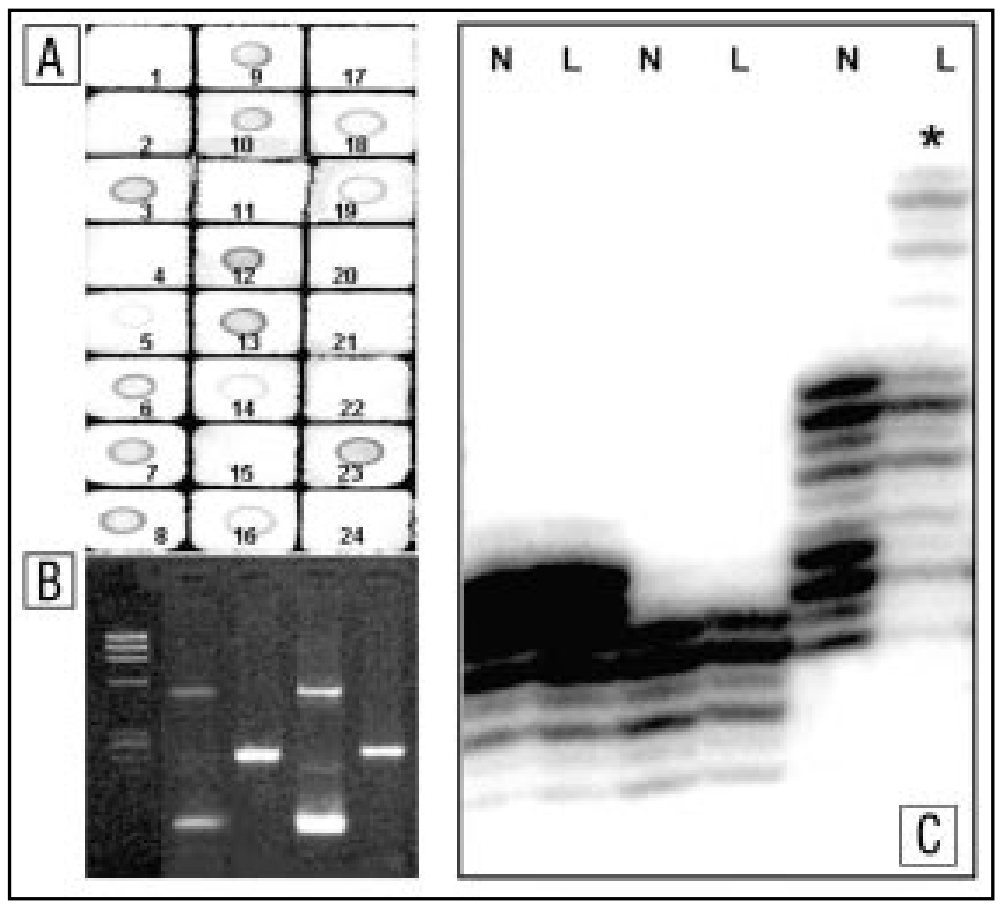

Figura 1. Tipificación de HPV mediante técnica combinada de PCR dot blot (A), RFLP (B) la determinación de inestabilidad microsatelital se realizó con PCR radioactivo y revelado mediante por autoradiografía. En la fotografía $(\mathrm{C})$ se observa controles internos $(\mathrm{N})$ y casos $(\mathrm{T})$ observando el la zona del asterisco $\left({ }^{*}\right)$ la presencia de bandas extras en el alelo superior del microsatélite estudiado, evidenciado inserciones que hacen que el producto de PCR migre menos en el gel debido al mayor peso molecular. 
Figura 2. Lesiones histológicas en cuello uterino. En la microfotografía compuesta se observa una lesión de bajo grado correspondiente a signos morfológicos de infección por HPV (A) una lesión de alto grado representada por un carcinoma in situ (B) y la imagen de un carcinoma espinocelular moderadamente diferenciado infiltrante (C).

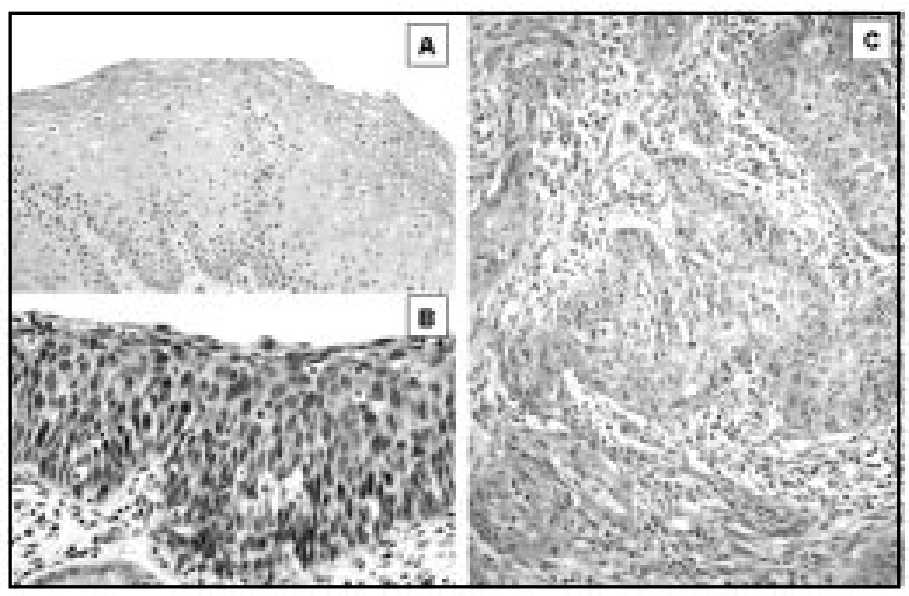

ción de genotipos virales 16/52 y 45/52 (Tabla 1 ).

De los marcadores de microsatélites estudiados, los localizados en los cromosomas 2, 12 y 16 fueron los más frecuentes (D2S123, D12S1638 y D16S265) alrededor de 20\% cada uno. Para el resto de los marcadores la frecuencia, fluctuó entre $4,5 \%$ y $15 \%$ y sólo el marcador D5S346 asociado al gen APC no mostró inestabilidad en ninguno de los casos examinados (Tabla 2).

$\mathrm{Al}$ analizar la frecuencia de inestabilidad microsatelital en al menos uno de los marcadores usados, se obtuvo $55,6 \%$, 90,9\% y $68,75 \%$ en los

Tabla 1 G enotipos de H PV según diagnóstico histológico

\begin{tabular}{|lcccccccccc|}
\hline Diagnóstico & & \multicolumn{1}{c}{ Genotipos Virales } \\
& $\mathrm{n}$ & 16 & 18 & 31 & 33 & 35 & 45 & 52 & $\mathrm{IM}^{*}$ & ARo \\
\hline Cancinoma & 28 & 9 & 1 & 0 & 4 & 0 & 2 & 1 & 0 & 11 \\
LAG & 22 & 2 & 0 & 2 & 10 & 1 & 2 & 1 & 4 & 0 \\
LBG & 16 & 3 & 0 & 4 & 4 & 2 & 0 & 0 & 1 & 0 \\
Total (\%) & 66 & $14(21,2)$ & $1(1,5)$ & $6(9,1)$ & $18(27,2)$ & $3(4,5)$ & $4(6,1)$ & $4(6,1)$ & $5(7,6)$ & $11(16,7)$ \\
\hline
\end{tabular}

$\mathrm{HPV}=$ Virus papiloma humano. $\mathrm{Ca}=$ Carcinoma invasor. $\mathrm{LAG}=$ Lesión de alto grado. LBG= Lesión de bajo grado. IM*= Infección mixta (4 casos HPV16/52 y un caso HPV 45/52). ARo= Alto riesgo (HPV 16, 18, 45 y 52), genotipificado por hibridación dot blot.

Tabla 2. M SI según microsatélite y diagnóstico histológico

\begin{tabular}{|lccccccccc|}
\hline & & \multicolumn{8}{c|}{ Inestabilidad microsatelital } \\
& $\mathrm{n}$ & BAT25 & BAT26 & D2S123 & D5S346 & D17S250 & D3S1067 & D12S1638 & D16S265 \\
\hline Carcinoma & 28 & 3 & 2 & 3 & 0 & 2 & 1 & 5 & 4 \\
LAG & 22 & 1 & 5 & 5 & 0 & 4 & 2 & 6 & 8 \\
LBG & 16 & 2 & 2 & 6 & 0 & 3 & 0 & 3 & 4 \\
Total (\%) & 66 & $8 / 66$ & $9 / 66$ & $13 / 66$ & & $9 / 66$ & $3 / 66$ & $14 / 66$ & $16 / 62$ \\
& & $(12,1)$ & $(13,6)$ & $(19,7)$ & 0 & $(13,6)$ & $(4,5)$ & $(21,2)$ & $(25,8)$ \\
\hline
\end{tabular}


casos de carcinomas invasor, LAG y LBG, respectivamente. Sin embargo, cuando utilizamos la clasificación propuesta por el NCI, sólo encontramos un pequeño porcentaje de los casos con inestabilidad microsatelital de alto grado (MSI-H) (4,5\%). El porcentaje de inestabilidad microsatelital de bajo grado (MSI-L) alcanzó 60\% y no se observó inestabilidad microsatelital en $36,5 \%$ de los casos. Ninguno de los casos con carcinoma invasor presentó MSI-H, los restantes casos se distribuyeron en forma similar. El alto porcentaje de casos de MSI-L observado no mostró diferencias significativas en los diferentes grupos de lesiones estudiados (Tabla 3).

Al tabular los diferentes subtipos de HPV según el grado de inestabilidad microsatelital, observamos que los subtipos virales 16, 31 y 33 resultaron estar más frecuentemente asociados con MSI-L Sin embargo, estas diferencias no fueron estadísticamente significativas (Tabla 4). Dos tercios de los pacientes en los cuales no fue posible tipificar el genotipo viral (es decir, probablemente un genotipo viral poco frecuente), no presentaron MSI.

\section{Discusión}

En la actualidad, los estudios sobre $\mathrm{CaCu}$ y HPV están focalizados sobre las alteraciones genéticas que generan una mayor susceptibilidad para que la célula hospedera sufra transformación maligna ${ }^{4,5,30}$. Entre ellas, se encuentran la deleción alélica ( $\mathrm{LOH}$ ) relacionada con inactivación de genes supresores de tumores y que ha sido descrita en la progresión de lesiones preneoplásicas. La deleción alélica más frecuente se ha observado en el brazo corto del cromosoma 3, como un evento precoz en la tumorogénesis cervical uterina ${ }^{22,31,32}$. También ha sido reportada MSI, en $\mathrm{CaCu}$ y lesiones preneoplásicas ${ }^{23,33}$, concordando con nuestros resultados, sin embargo, otros autores, como Nishimura M et al (2000), concluyeron, a partir de sus resultados, que la inestabilidad genómica es un evento tardío durante la carcinogénesis del $\mathrm{CaCu}$ y se asocia con la progresión de una neoplasia cervical intraepitelial a un carcinoma invasor. En nuestro trabajo no analizamos la presencia de $\mathrm{LOH}$ debido a que, a diferencia de la subtipificación HPV y determina-

Tabla 3. Grado M SI según diagnóstico histológico

\begin{tabular}{|lccc|}
\hline & & Grados de MSI $(\%)$ & \\
& MSI-H & MSI-L & MSS \\
\hline Carcinoma & 0 & $13(46)$ & $3(54)$ \\
LAG & $2(9,1)$ & $17(77,2)$ & $3(13,7)$ \\
LBG & $1(6,3)$ & $9(56,2)$ & $6(37,5)$ \\
Total (\%) & $3(4,5)$ & $39(59)$ & $24(36,5)$ \\
\hline
\end{tabular}

Tabla 4. Grado de MSI según genotipo de H PV

\begin{tabular}{|lccccccccc|}
\hline & & & \multicolumn{9}{c}{ Tipos de HPV } & & & & \\
& $\mathrm{n}$ & 16 & 18 & 31 & 33 & 35 & 45 & 52 & $\mathrm{AR}^{\circ}$ \\
\hline MSI-H & 3 & 0 & 0 & 0 & 0 & 0 & 0 & 2 & 1 \\
MSI-L & 39 & 9 & 1 & 5 & 14 & 3 & 4 & 0 & 4 \\
MSS & 24 & 8 & 0 & 1 & 4 & 0 & 0 & 0 & 10 \\
MSI/ total & & $9 / 17(53 \%)$ & $1 / 1$ & $5 / 6(83)$ & $14 / 18(78)$ & $3 / 3$ & $4 / 4$ & $2 / 2$ & $5 / 15(34)$ \\
\hline
\end{tabular}

$\mathrm{MSI}-\mathrm{H}=\mathrm{MSI}$ alta frecuencia. MSI-L= MSI baja frecuencia. $\mathrm{AR}^{\circ}=$ Alto riesgo (HPV 16, 18, 45 у 52), genotipificado por hibridación dot blot. 
ción de MSI, ésta requiere pureza celular que sólo se obtiene mediante microdisección neumática 0 láser.

En relación a MSI y genotipos de HPV en LBG, LAG y carcinomas invasores de cuello uterino, existe escasa información publicada y no existe nomenclatura uniforme para la determinación y clasificación de MSI en los reportes publicados sobre genotipos específicos de HPV. Este hecho es particularmente desorientador, porque impide hacer las comparaciones necesarias que permitan extrapolar conclusiones. En nuestro estudio utilizamos la nomenclatura y clasificaciones actualmente aceptadas para otros órganos, como cáncer de colon, estómago y endometrio, encontrando una baja frecuencia de MSI-H en los casos analizados $(4,5 \%)$ y considerablemente alta de MSI-L y MSS (60\% y 35,5\%, respectivamente). Los marcadores propuestos por el NCI junto a otro panel de marcadores (BAT40, D3S1263, D4S402, D5S406 y D11S912), fueron empleados por Wong et $\mathrm{al}^{34}$ para el análisis de MSI en neoplasia cervical. Combinando ambos paneles, estos autores reportaron $29 \%$ de MSI en los casos de $\mathrm{CaCu}$ analizados, siendo la frecuencia de MSI en los carcinomas significativamente más alta que en LAG y LBG.

Otros estudios han encontrado MSI-H en carcinomas con frecuencias entre $14 \%$ y $18,8 \% 3,31,35$. Chung et $\mathrm{al}^{3}$ encontraron MSI-H en $14 \%$ de los casos de carcinoma y concluyeron que la inestabilidad microsatelital era un evento raro en la carcinogénesis cervical y que es independiente de la presencia de HPV. Wong et $\mathrm{al}^{34}$ encontraron MSI-L en menos de $2 \%$ de las lesiones de alto grado y un MSI-H en 11,8\% de los casos de $\mathrm{CaCu}$, encontrando asociación con peor pronóstico en aquellos pacientes con algún grado de MSI. También ha sido reportado $17,6 \%$ de MSI en casos HPV positivos (sólo para los genotipos 16, 18 y 33) y $7,9 \%$ en casos HPV negativos ${ }^{31}$. Narayan et $\mathrm{al}^{35}$ encontraron $18,8 \%$ de MSI-H en tumores primarios HPV positivos. French et $\mathrm{al}^{33}$ estudiaron 32 lesiones escamosas del cuello uterino con una metodología y nomenclatura diferentes, considerando 7 microsatélites diferentes a los nuestros, no encontrando MSI-H ni relación con los 4 genoti- pos virales $(6,11,16$ y 18$)$ que consideraron en su estudio.

Los porcentajes de MSI-L fluctuaron entre 12\% y $47 \%$ de los casos en otras series ${ }^{3,33}$. Al combinar los casos de alto y de bajo grado de inestabilidad, no se observó significativas diferencias en el pronóstico y conducta biológica de los tumores, sin embargo, se observó una leve tendencia a menor sobrevida en aquellos pacientes que presentaban inestabilidad de alto grado ${ }^{3}$. Es particularmente interesante que los virus de alto riesgo oncogénico presenten la mayor frecuencia de MSS y menor de MSI-H, una probable explicación en este punto podría radicar en que las vías utilizadas por los virus oncogénicos no requieren de MSI, la cual si sería más importante para los virus de menor potencial oncogénico.

Por otro lado, la presencia de MSI en lesiones preneoplásicas o cánceres y su relación con el pronóstico de la patología quedan aún por establecerse.

Es conocido que la inflamación crónica tiene un impacto en la capacidad reparadora del ADN desalineado, cuya alteración se relaciona directamente con la aparición de inestabilidad microsatelital debido a inactivación parcial total del sistema reparador de ADN desalineado, principalmente por mecanismos de metilación ${ }^{36}$. Esto plantea la necesidad de definir la importancia de la cervicitis crónica inespecífica prolongada y fenómenos epigenéticos en la génesis del cáncer de cuello uterino mediada por inestabilidad genética localizada.

Cuando analizamos la frecuencia de subtipos virales con la presencia de MSI de bajo grado observamos tendencia a mayor acumulación con los subtipos 16, 31, 33, sin embargo, estas diferencias no fueron estadísticamente significativas. En resumen, podemos decir que en casi dos tercios de los casos presentaron MSI-L, lo que demuestra la participación de inestabilidad genética desde las primeras etapas de la tumorogénesis cervical uterina. La baja frecuencia MSI-H y su agregación específica con el HPV52 y la menor frecuencia de MSI-L en casos HPV16, sugieren mecanismos coadyuvantes diferentes en la carcinogénesis cervical uterina mediada por virus de alto riesgo oncogénico. 


\section{REFERENCIAS}

1. Bosch FX, Manos MM, Munoz N, Sherman M, Jansen AM, Peto J et aL. Prevalence of human papillomavirus in cervical cancer: a perspective. International biological study on cervical cancer Study Group. J Natl Cancer Inst 1995; 87: 796-802.

2. VIша LL. Human papillomaviruses and cervical cancer. Adv Cancer Res 1997; 71: 321-41.

3. Chung TK, Cheung TH, Wang VW, Yu MY, Wong YF. Microsatellite instability, expression of hMSH2 and hMLH1 and infection in cervical cancer and their clinico-pathological. Gynecol Obstet Invest 2001; 52: 98-103.

4. Clarke B, Gordon M, Moodley M, Naidoo R, CHETTY R. Microsatellite analysis of early stage (IaIIb) uterine cervical carcinoma. Int J Surg Pathol 2003; 11: 253-60.

5. WANI K, NAIR CK. Genetic alterations in cervical cancer. Indian J Exp Biol 2003; 41: 789-96.

6. BenNetT P. Demystified... microsatellites. Mol Pathol 2000; 53: 177-83.

7. Eshieman JR, Lang EZ, Bowerfind GK, Parsons R, Vogeistein B, WiLSON JK ET AL. Increased mutation rate at the hprt locus accompanies instability in colon cancer. Oncogene 1995; 10: 33-7.

8. Thibodeau SN, Bren G, Schaid D. Microsatellite instability in cancer of the proximal colon. Science 1993; 260: 816-9.

9. Helland A, Borresen-Daie Al, Peltomaki P, Hektoen M, Kristensen GB, NesLand JM et al. Microsatellite instability in cervical and endometrial. Int J Cancer 1997; 70: 499-501.

10. Rhyu MG, Park WS, Meltzer SJ. Microsatellite instability occurs frequently in human gastric. Oncogene 1994; 9: 29-32.

11. Roa JC, Araya JC, Viliseca Ma, Roa I, Correa P. [Microsatellite instability and loss of heterozygosity in preneoplastic gastric lesions]. Rev Méd Chile 2003; 131: 1227-36.

12. Nakata B, Wang YQ, Yashiro M, Nishioka N, Tanaka $H$, Ohira M et al. Prognostic value of microsatellite instability in resectable cancer. Clin Cancer Res 2002; 8: 2536-40.

13. Abraham SC, Wu TT, Hruban RH, Lee JH, Yeo CJ, ConLON K ET AL. Genetic and immunohistochemical analysis of pancreatic acinar carcinoma: frequent allelic loss on chromosome 11p and APC/ beta-catenin pathway. Am J Pathol 2002; 160: 953-62.
14. MerLo A, Mabry M, Gabrielson E, Volmer R, Baylin SB, SIDRANSKY D. Frequent microsatellite instability in primary small cell lung. Cancer Res 1994; 54: 2098-101.

15. Takahashi $Y$, Kondo $K$, Hirose $T$, Nakagawa $H$, Tsuyuguchi M, Hashimoto M et al. Microsatellite instability and protein expression of the DNA repair gene, hMLH1, of lung cancer in chromateexposed workers. Mol Carcinog 2005; 42: 150-8.

16. Risinger Ji, Berchuck A, Kohler MF, Watson P, Lynch HT, Boyd J. Genetic instability of microsatellites in endometrial carcinoma. Cancer Res 1993; 53: 5100-3.

17. Kaneki E, Oda Y, Ohishi Y, Tamiya S, Oda S, HiraKawa T et al. Frequent microsatellite instability in synchronous ovarian and adenocarcinoma and its usefulness for differential diagnosis. Hum Pathol 2004; 35: 1484-93.

18. Hirasawa A, Aoki D, Inoue J, Imoto I, Susumu N, Sugano K ET aL. Unfavorable prognostic factors associated with high frequency of microsatellite instability and comparative genomic hybridization in endometrial cancer. Clin Cancer Res 2003; 9: 5675-82.

19. King BL, Carcangiu ML, Carter D, Kiechle M, Pfisterer J, Pfieiderer A et al. Microsatellite instability in ovarian neoplasms. Br J Cancer 1995; 72: 376-82.

20. Singer G, Kawnowski T, Hartmann A, Dietmaier W, WiLd PJ, Schraml P ET al. Different types of microsatellite instability in ovarian. Int J Cancer 2004; 112: 643-6.

21. Delias A, Puhl A, Schraml P, Thomke SE, Ruschoff J, Minatsch MJ et al. Molecular and clinicopathological analysis of ovarian carcinomas without microsatellite instability. Anticancer Res 2004; 24: 361-9.

22. Kersemaekers aM, Kenter GG, Hermans J, Fleuren GJ, vAN DE VIJver MJ. Allelic loss and prognosis in carcinoma of the uterine cervix. Int J Cancer 1998; 79: 411-7.

23. Jiménez P, Canton J, Concha A, Torres LM, Abril E, REAL LM ET AL. Microsatellite instability in cervical intraepithelial. J Exp Clin Cancer Res 1998; 17: 361-6.

24. Saiki RK, Scharf S, Faloona F, MuLis KB, Horn GT, ERLCH HA ET AL. Enzymatic amplification of betaglobin genomic sequences and site analysis for diagnosis of sickle cell anemia. Science 1985; 230: 1350-4. 
25. Manos MM TY, Wright DK ET AL. Use of polymerase chain reaction amplification for the detection of genital human papillomaviruses. Cancer Cells 1989; 7: 209-14.

26. Jacobs MV, Snijders PJ, van den Brule AJ, Helmerhorst TJ, Mejer CJ, Walboomers JM. A general primer GP5+/GP6(+)-mediated PCR-enzyme immunoassay rapid detection of 14 high-risk and 6 low-risk human genotypes in cervical scrapings. J Clin Microbiol 1997; 35: 791-5.

27. Snijders PJ, van den Brule AJ, Schrjnemakers HF, Snow G, Mejer CJ, Walboomers JM. The use of general primers in the polymerase chain reaction detection of a broad spectrum of human papillomavirus genotypes. J Gen Virol 1990; 71: 173-81.

28. Melo A, Montenegro S, Hooper T, Capurro I, Roa J, RoA I. Tipificación del virus papiloma humano (VPH) en lesiones preneoplásicas y carcinoma del cuello uterino en mujeres de la IX regiónChile. Rev Méd Chile 2003; 131: 1382-90.

29. Boland CR, Thibodeau SN, Hamilton SR, Sidransky D, Eshieman JR, Burt RW et al. A National Cancer Institute Workshop on Microsatellite cancer detection and familial predisposition: development of criteria for the determination of microsatellite instability in cancer. Cancer Res 1998; 58: 5248-57.

30. Wong YF, Selvanayagam ZE, Wei N, Porter J, Vittal R, Hu R ET AL. Expression genomics of cervical cancer: molecular classification prediction of radiotherapy response by DNA microarray. Clin Cancer Res 2003; 9: 5486-92.

31. Kohno T, Takayama $H$, Hamaguchi M, Takano $H$, Yamaguchi N, Tsuda $H$ et al. Deletion mapping of chromosome $3 p$ in human uterine cervical cancer. Oncogene 1993; 8: 1825-32.

32. Cheung TH, Lo KW, Yim SF, Poon CS, Cheung AY, CHUng TK ET AL. Clinicopathologic significance of loss of heterozygosity on in cervical cancer. Gynecol Oncol 2005; 96: 510-5.

33. French D, Cermele C, Vecchione A, Cenci M. HPV infection and microsatellite instability in squamous lesions uterine cervix. Anticancer Res 2000; 20: 3417-21.

34. Wong YF, Cheung TH, Poon KY, Wang VW, Li JC, Lo KW ET AL. The role of microsatellite instability in cervical neoplasia and squamous cell carcinoma of the cervix. Gynecol Oncol 2003; 89: 434-9.

35. Narayan G, arias-Pulddo H, Koul S, Vargas H, Zhang FF, ViLelia J Et al. Frequent promoter methylation of CDH1, DAPK, RARB, and HIC1 carcinoma of cervix uteri: its relationship to clinical outcome. Mol Cancer 2003; 2: 24.

36. Rashid A, Ueki T, Gao YT, Houlhan PS, WaLace C, WANG BS ET AL. K-ras mutation, p53 overexpression, and microsatellite instability in biliary tract cancers: a population-based study in China. Clin Cancer Res 2002; 8: 3156-63. 